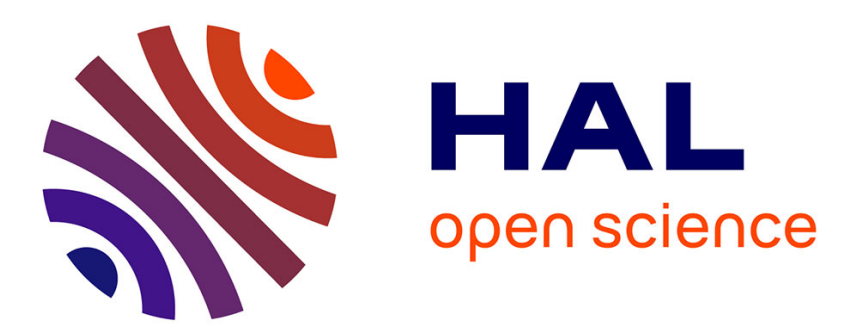

\title{
Coalescence times, life history traits and conservation concerns: An example from four coastal shark species from the Indo-Pacific
}

Pierre Lesturgie, Serge Planes, Stefano Mona

\section{- To cite this version:}

Pierre Lesturgie, Serge Planes, Stefano Mona. Coalescence times, life history traits and conservation concerns: An example from four coastal shark species from the Indo-Pacific. Molecular Ecology Resources, 2021, 10.1111/1755-0998.13487 . hal-03359531

\section{HAL Id: hal-03359531 \\ https: / hal.sorbonne-universite.fr/hal-03359531}

Submitted on 30 Sep 2021

HAL is a multi-disciplinary open access archive for the deposit and dissemination of scientific research documents, whether they are published or not. The documents may come from teaching and research institutions in France or abroad, or from public or private research centers.
L'archive ouverte pluridisciplinaire $\mathbf{H A L}$, est destinée au dépôt et à la diffusion de documents scientifiques de niveau recherche, publiés ou non, émanant des établissements d'enseignement et de recherche français ou étrangers, des laboratoires publics ou privés. 


\section{MOLECULAR ECOLOGY RESOURCES}

\section{Coalescence times, life history traits and conservation concerns: an example from four coastal shark species from the Indo-Pacific}

\begin{tabular}{|r|l|}
\hline Journal: & Molecular Ecology Resources \\
\hline Manuscript ID & MER-21-0182.R1 \\
\hline Manuscript Type: & Resource Article \\
\hline Date Submitted by the \\
Author: & 27-Jul-2021 \\
\hline Complete List of Authors: & $\begin{array}{l}\text { Lesturgie, Pierre; Museum National d'Histoire Naturelle, Origines et } \\
\text { Evolution } \\
\text { Planes, Serge; Université de Perpignan, UMR 5244 CNRS-EPHE-UPVD; } \\
\text { CRIOBE, UMS 2978 } \\
\text { Mona, Stefano; Museum National d'Histoire Naturelle, Origines et } \\
\text { Evolution; EPHE PSL }\end{array}$ \\
\hline Keywords: & $\begin{array}{l}\text { Coalescence, Population Genomics, Meta-Population, Sharks, Life History } \\
\text { Traits }\end{array}$ \\
\hline &
\end{tabular}


1 Coalescence times, life history traits and conservation concerns: an example from four 2 coastal shark species from the Indo-Pacific

3

4 Running title: Coalescence times and conservation concerns.

5

6

7 Pierre Lesturgie ${ }^{1}$, Serge Planes ${ }^{2,4}$, Stefano Mona ${ }^{1,2,3, *}$

8

$9 \quad{ }^{1}$ Institut de Systématique, Evolution, Biodiversité, ISYEB (UMR 7205), Muséum National

10 d'Histoire Naturelle, CNRS, Sorbonne Université, EPHE, Université des Antilles, Paris,

11 France.

$12 \quad{ }^{2}$ EPHE, PSL Research University, Paris, France.

$13{ }^{3}$ Laboratoire d'Excellence CORAIL, Papetoai, French Polynesia.

$14{ }^{4}$ PSL Research University: EPHE-UPVD-CNRS, USR 3278 CRIOBE, Université de

15 Perpignan, 52 Avenue Paul Alduy, 66860, Perpignan, Cedex, France

16

17

$18 *$ Corresponding author. E-mail : stefano.mona@mnhn.fr 


\section{Abstract}

Dispersal abilities play a crucial role in shaping the extent of population genetic structure, with more mobile species being panmictic over large geographic ranges and less mobile ones organized in meta-populations exchanging migrants to different degrees. In turn, population structure directly influences the coalescence pattern of the sampled lineages, but the consequences on the estimated variation of the effective population size $(\mathrm{Ne})$ over time obtained by means of unstructured demographic models remain poorly understood. However, this knowledge is crucial for biologically interpreting the observed $\mathrm{Ne}$ trajectory and further devising conservation strategies in endangered species. Here we investigated the demographic history of four shark species (Carharhinus melanopterus, Carharhinus limbatus, Carharhinus amblyrhynchos, Galeocerdo cuvier) with different degrees of endangered status and life history traits related to dispersal distributed in the Indo-Pacific and sampled off New Caledonia. We compared several evolutionary scenarios representing both structured (meta-population) and unstructured models and then inferred the Ne variation through time. By performing extensive coalescent simulations, we provided a general framework relating the underlying population structure and the observed $\mathrm{Ne}$ dynamics. On this basis, we concluded that the recent decline observed in three out of the four considered species when assuming unstructured demographic models can be explained by the presence of population structure. Furthermore, we also demonstrated the limits of the inferences based on the sole site frequency spectrum and warn that statistics based on linkage disequilibrium will be needed to exclude recent demographic events affecting meta-populations. 


\section{Introduction}

41

42
Reconstructing the evolutionary history of a species is a challenging exercise only partially eased by the growing size of genetic data available. Indeed, larger amounts of data will provide more precision but not more accuracy if the model(s) chosen to infer demographic parameters is distant from the true one. Species are dynamic entities whose geographic range has often changed in time through range expansions, contractions and shifts (Arenas, Ray, Currat, \& Excoffier, 2012; Excoffier, Foll, \& Petit, 2009; Mona, Ray, Arenas, \& Excoffier, 2014). As a consequence, many species are most likely organized in meta-populations (i.e. groups of demes or sub-populations exchanging migrants to some extent), even though the more vagile ones might be panmictic at a large scale (Corrigan et al., 2018; Karl et al., 2010). Neglecting the meta-population structure (i.e., performing demographic inferences under unstructured models) may lead to spurious inference of population size change (Chikhi, Sousa, Luisi, Goossens, \& Beaumont, 2010; Maisano Delser et al., 2019, 2016; Mazet, Rodríguez, \& Chikhi, 2015), which is particularly worrisome for species of conservation concern. Unfortunately, the link between the inferred temporal trajectory of the effective population size $(\mathrm{Ne})$ and the real demographic history of the meta-population remains largely under explored. However, the role of connectivity, particularly the number of migrants $\mathrm{Nm}$ exchanged each generation and the migration matrix, has been put forward as a key actor in shaping the gene genealogy of lineages sampled from a deme belonging to a meta-population (Chikhi et al., 2010; Mona et al., 2014; Ray, Currat, \& Excoffier, 2003; Städler, Haubold, Merino, Stephan, \& Pfaffelhuber, 2009). Understanding the relations between meta-population structure, the inferred $\mathrm{Ne}$ variation under unstructured models, and species dispersal abilities, is crucial to correctly interpret the pattern of genetic variability and to establish conservation priorities. To search for general rules describing such relations, we followed an inductive approach investigating species: i) with large 
64 distribution (which in principle should guarantee an organization in meta-populations); ii) with different life history traits (LHT) related to dispersal; iii) of conservation concerns. In this spirit, we selected for our genomic study four shark species (Carcharhinus amblyrhynchos, Carcharhinus limbatus, Carcharhinus melanopterus, and Galeocerdo cuvier) from New Caledonia. These species have a large and overlapping distribution in the Indo-Pacific (https://sharksrays.org/) and they differ for LHT features such as size (which is positively correlated with the capacity for long distance swimming and oceanic migration (Parsons, 1990)), residency pattern, and long-distance dispersal ability as measured by tagging data (Table S1). Moreover, the IUCN red list reported that the black-tip shark (C. limbatus) and the tiger shark (G. cuvier) are Near Threatened (with a decreasing trend in the tiger shark), the black-tip reef shark (C. melanopterus) is Vulnerable with decreasing trend, and the grey reef shark (C. amblyrhynchos) is Endangered with decreasing trend as well. We first compared several population genetics models by means of coalescent simulations coupled with an approximate Bayesian computation framework (Bertorelle, Benazzo, \& Mona, 2010) to detect whether panmixia or a meta-population model best describe the genomic variation of each species. Then, we inferred the demographic parameters under the most likely model and applied the stairwayplot, which assumes a panmictic unstructured population (Liu \& Fu, 2015), to detect the $N e$ variation through time in each species. We finally run extensive coalescent simulations under the tested meta-population models with parameters compatible to those observed in real data. The simulated datasets were in turn analysed with the stairwayplot to: i) help interpreting the observed data in the four shark species; ii) providing general coalescence arguments relating the demographic history of a meta-population and the reconstructed variation in Ne through time by means of unstructured models. 


\section{$88 \quad$ Material \& Methods}

89 Sampling

90 Eight specimens of tiger shark (G. cuvier), 13 black tip shark (C. limbatus), and 12 grey reef 91 shark (C. amblyrhynchos) were collected off New Caledonia. Total genomic DNA was 92 extracted from muscle tissue or fin clips, and preserved in 96\% ethanol using QIAGEN DNeasy 93 Blood and Tissue purification kit (Qiagen, Hilden, Germany) according to the manufacturer's 94 protocols. Double-digest restriction-associated DNA (ddRAD) libraries were prepared 95 following Peterson, Weber, Kay, Fisher and Hoekstra (2012) using EcoRI and MspI restriction 96 enzymes and a 400-bp size selection. The genomic libraries obtained were sequenced with a 97 HiSeq 2500 Illumina sequencer (single-end, $125 \mathrm{bp}$ ). Exon capture data of eight $C$. melanopterus) from New Caledonia (Maisano Delser et al., 2019) were included in this study

99 for comparative purposes.

\section{De novo assembly and data filtering (dd-RADseq samples)}

101 Raw reads were first demultiplexed and quality filtered through the process_radtags.pl pipeline 102 in Stacks v.2.5 (Rochette, Rivera-Colón, \& Catchen, 2019). In the absence of a reference 103 genome for any of the three species, RAD-seq loci were de novo assembled independently in each species under the denovo_map.pl pipeline in Stacks. We used the following assembly parameters: $m=3$ (minimum read depth to create a stack), $M=4$ (number of mismatches allowed between loci within individuals), and $n=4$ (number of mismatches allowed between loci within

107 catalogue). We found an average coverage per species of $\sim 10 \mathrm{x}$ (see results). A consensus on 108 the threshold below which SNP calling may be considered unreliable is still lacking. However, 109 genotype free estimation of allele frequency is generally recommended with low to medium 110 coverage (Korneliussen, Albrechtsen, \& Nielsen, 2014). This approach, implemented in the 111 software Angsd v.0.923 (Korneliussen et al., 2014), has been rarely applied to Rad-seq data 
112 (however, see Warmuth and Ellegren (2019) for an exception) and, to our knowledge, never to

113 Rad-seq data from non-model organisms, probably due to the need of a reference sequence for

114 the software to work. Here, we followed the approach of Heller et al. (2021) and Khimoun et

115 al. (2020) by creating an artificial reference sequence. First, we used the population script in

116 Stacks to assemble loci present in at least $80 \%$ of the individuals (using the flag $r=0.8$ ); then,

117 we concatenated the consensus sequences of the retrieved loci spaced by a stretch of $120 \mathrm{~N}$

118 (unknown) characters (the same length of the Rad-loci) to facilitate the subsequent mapping.

119 Raw reads were then mapped back to the novel reference sequence by means of the bwa-mem

120 algorithm with default parameters (Li \& Durbin, 2009). Using custom bash scripts coupled with

121 Angsd, we applied a number of filters to the aligned data and eliminated: $i$ ) sites with coverage

$122<3$ (-minIndDepth=3 flag), ii) bad quality bases and poorly aligning reads (-min $Q$ and -

123 minMap $Q$ and $-C$ flags with default values); iii) poor quality sites based on the per base

124 alignment quality (-baq=1 flag); $i v)$ SNPs in the last 5 bp of each locus; $v$ ) SNPs heterozygote

125 in at least $80 \%$ of individuals; vi) loci with more than 5 SNPs that could potentially be

126 paralogous; vii) sites with missing data by setting the -minInd flag to the total number of

127 individuals retained in each species. The filtered dataset was then used to generate a site allele

128 frequency likelihood file, with the genotype likelihoods computed with the SAMtools method

$129(-G L=1$ flag), further optimised to compute a folded site frequency spectrum (SFS) with no

130 missing data for downstream analyses. An alternative (and simpler) approach would have been

131 to augment $m$ to achieve an higher coverage (Paris, Stevens, \& Catchen, 2017). However,

132 beside the considerable loss in the number of assembled loci (and hence of retrieved SNPs), we

133 found by extensive simulation of in silico Rad experiments that selecting high coverage loci

134 biases the SFS towards low frequency variants (Mona, Bertorelle, Benazzo, in preparation). 
135 The SFS for C. melanopterus was estimated directly from the high coverage exon-capture 136 dataset of Maisano Delser et al. (2019).

\section{Genetic diversity and demographic inferences}

138 Nucleotide diversity $\left(\theta_{\pi}\right), \theta_{w}$ (Watterson's theta, based on segregating sites (Watterson, 1975)) 139 and Tajima's $D(T D$, (Tajima, 1989)) were computed from the SFS for each species with custom 140 scripts. Significance of $T D$ was evaluated after 1,000 coalescent simulations of a constant 141 population model with scaled size $\theta_{\pi}$. To test whether sampled demes are isolated or belong to 142 a structured meta-population and to eventually estimate connectivity, we devised three 143 alternative evolutionary models for each species (Figure 1) within an approximate bayesian 144 computation (ABC) framework. Model NS (non-structured) defined an isolated population 145 characterized by a modern effective population size $\left(N_{M O D}\right)$ switching instantaneously into an 146 ancestral population size $\left(N_{A N C}\right)$ at $T c$ generations before present. Model FIM specifies a non147 equilibrium finite island model defined by $d=100$ demes exchanging $N m$ migrants each 148 generation under a symmetric migration matrix. The array of demes is instantaneously 149 colonized $T_{C O L}$ generations before present from a population with an ancestral size $\left(N_{A N C}\right)$. 150 Model SST is similar to FIM but demes exchange migrants only with their four neighbours (or 151 less, if they are at the border of the array), in a steppingstone fashion. We performed 50,000 152 coalescent simulations from prior distributions using fastsimcoal v.2.6.0.3 (Excoffier, 153 Dupanloup, Huerta-Sánchez, Sousa, \& Foll, 2013), reproducing the exact number of individuals 154 and loci for each species (Table 1). We first performed model selection through the random 155 forest (RF) classification method implemented in the abcRF R package (Pudlo et al., 2016). We 156 then performed 50,000 additional simulations under the most supported model in order to 157 estimate demographic parameters with the abcRF regression method (Raynal et al., 2019). Both 158 model selection and parameter estimation were computed with the following set of summary 
159 statistics: the SFS, $\theta_{\pi}, \theta_{w}$ and $T D$. The first two axes of a Linear Discriminate Analysis

160 performed on the previous statistics were also included for model selection in order to increase

161 the accuracy of the estimates (Pudlo et al., 2016). Even though $\theta_{\pi}, \theta_{w}$ and TD are function of

162 the SFS, they convey additional information by the non-linear feature of the functions.

163 Information redundancy among the considered summary statistics is accounted for by the RF

164 algorithm. Model selection and parameter estimation were run twice on each set of simulations

165 to check the consistency of the analyses, and cross validation (or confusion matrix for the model

166 selection) was performed on the first of the two runs. The number of trees in each RF algorithm

167 was chosen by monitoring the evolution of the out-of-bag error (Pudlo et al., 2016).

168 We investigated the variation in the effective population size (Ne) through time by running the

169 composite likelihood approach implemented in the stairwayplot v.0.2 software (Liu \& Fu,

170 2015). We set the generation time to seven years for C. melanopterus (Maisano Delser et al.,

1712016 ) and to 10 years for the other species (Cortés, 2002; Pirog et al., 2019) for all demographic

172 inferences. We applied a mutation rate per generation per site of $8.4 \times 10^{-9}$ to the exon capture

173 data of C. melanopterus (Maisano Delser et al. 2016) and of $1.93 \times 10^{-8}$ to the RADseq data for

174 the remaining three species. This mutation rate was determined by scaling genetic diversity

175 between ddRAD (obtained under the same protocol of this study) and Exon Capture data from

17612 C. melanopterus individuals from Moorea, French Polynesia (Supplementary Material).

\section{Simulation study}

178 We ran coalescent simulations under FIM, SST and their modified version FIM-CH and SST-

$179 \mathrm{CH}$, where the $\mathrm{Nm}$ parameter is changed at $T_{C H}$ generations B.P. (Figure 1), to first inspect the

180 shape of the SFS and to further uncover the variation of $N e$ over time assuming a panmictic

181 population by means of the stairwayplot. We investigated in total 288 demographic scenarios

182 under the four meta-population models (Tables 2, S2, S3, S4, S5 and S6). Similarly to the 
183 analyses performed on the real data, all scenarios were represented by $d=100$ demes exchanging 184 migrants. We sampled 10 diploid individuals either from a randomly selected deme in the case 185 of FIM/FIM-CH (since all demes have the same coalescence history) or from the central deme 186 of the array in the case of SST/SST-CH (to avoid border effects). Deme size was fixed to $N_{D E M E}$ $187=5000$ with $m$ varying accordingly to obtain a long-term $N m$ of $1,5,10$, and 15 in order to 188 encompass the range of the estimated values (see results). $T_{C O L}$ was fixed to 5,000, 15,000 and 18950,000 generations B.P. or to $\infty$ (i.e., equilibrium model), and the ancestral effective size was 190 fixed to $N_{A N C}=50,000$. Change of connectivity occurred at $T_{C H}=10$ or 50 generations B.P., to 191 mimic human induced effects due to overfishing and/or habitat modifications (i.e., climate 192 changes). Looking forward in time, we modelled the change in connectivity by instantaneously 193 decreasing $m$ or $N_{D E M E}$ by a factor 10 or 100 with respect to the long-term $N m$ (Tables S3, S4,

194 S5 and S6). For each combination of parameters, we performed 100 coalescent simulations of 19550,000 Rad-like loci of $115 \mathrm{bp}$. Mutation rate per site per generations was set to $1.93 \times 10^{-8}$ and 196 the generation time to 10 years. We computed for each scenario (averaged over the 100 197 replicates): a) summary statistics $\left(\theta_{\pi}, \theta_{w}\right.$, and TD); b) the normalised SFS as in (Lapierre, 198 Lambert, \& Achaz, 2017); c) the stairwayplot, to reconstruct the apparent variation of $\mathrm{Ne}$ 199 through time. We note that the number of diploid individuals and simulated loci were chosen 200 to be consistent with our data (preliminary analyses conducted on a subsample of 5,000 loci 201 produced consistent results).

\section{Results}

204 Summary statistics (number of assembled loci, SNPs, genetic diversity and Tajima's $D$ ) are 205 presented in Table 1. Mean coverage (and standard deviation) per sample was $9.02( \pm 2.62)$, $2067.93( \pm 0.48), 8.39( \pm 0.81)$ for G. cuvier, C. limbatus and C. amblyrhynchos respectively. 
207 We compared the models NS, FIM, and SST (Figure 1) in the four species by means of an 208 ABC-RF algorithm and estimated demographic parameters for the most supported model. After 209 checking for the evolution of the out-of-bag error of the RF, model selection and parameter 210 estimation were computed using respectively 500 and 1,000 trees in each species. We found 211 that NS had the higher posterior probability $(p=0.84)$ for G. cuvier (Tables 1 and S7). In 212 contrast, demographic histories of the three other species were best described by SST, with a 213 posterior probability ranging from 0.53 to 0.88 (Tables 1 and S7). The estimated median 214 number of migrants per generation $\mathrm{Nm}$ was 1.8 (95\% CI: 0.7-3.0) for C. melanopterus, $6.6(95 \%$ 215 CI: 1.5-15.4) for C. limbatus, and 11.5 (95\% CI: 3.0-22.0) for C. amblyrhynchos (Figure 2, 216 Table 1). The posterior distribution of $\mathrm{Nm}$ strongly differed from the prior distribution and 217 showed a clear unimodal peak with small credible intervals, and low mean square error (SME) 218 and mean root square error (SMRE) in all three species (Figure 2, Table S8, suggesting that 219 these estimates are highly reliable. Conversely, both $T_{C O L}$ and $N_{A N C}$ had larger SME and SMRE 220 errors in all species (Table S8), but it was only in C. melanopterus where posterior and prior 221 distribution could not be distinguished (Figure 2). $T_{C O L}$ has a clear unimodal distribution in $C$. 222 amblyrhynchos but a more disperse one (and with wider credible intervals) in C. limbatus 223 (Figure 2, Table 1).

224 The stairwayplot showed a nearly similar dynamic for C. amblyrhynchos and C. limbatus, 225 characterized by a strong ancestral expansion (Figure 3 ). When approaching $\mathrm{T}=0$, both species 226 underwent a bottleneck but of distinct strength. This is consistent with the shape of the 227 normalized SFS, which clearly shows a stronger deficit in low frequency variants for $C$. 228 limbatus compared to C. amblyrhynchos (Figure 3). Similarly to C. limbatus, C. melanopterus 229 experienced a recent 10 -fold population collapse around 20,000 years B.P. starting from a long 230 term constant Ne. However, C. melanopterus showed no signature of ancestral expansion, 
231 consistent to the results obtained by Maisano Delser et al. (2019) using abc-skyline method.

232 Finally, G. cuvier displayed an ancestral expansion around 100,000 years B.P. with $\mathrm{Ne}$ reaching

$233 \sim 12,000$ before dropping to $\sim 3000$ at $\mathrm{T} \sim 1,600$ years B.P. Remarkably, the ancestral expansion

234 retrieved by the stairwayplot (Figure 3) for both C. amblyrhynchos and C. limbatus overlap

235 with the posterior distribution of $T_{C O L}$ estimated by the SST model (Table 1). This analogy

236 holds too for C. melanopterus, where $T_{C O L}$ could not be properly estimated under the structured

237 model (we obtained a flat posterior distribution, Figure 2) and there was no signature of

238 ancestral expansion in the stairwayplot (Figure 3).

239 The first set of coalescent simulations was run under FIM and SST only (Table 2 and S2 to 240 check if simulated data could reproduce the pattern of genetic variability (both $\theta$ estimators and $241 T D$ ) observed for C. melanopterus, C.limbatus, and C. amblyrynchos. The simulated $\theta$ values

242 (excluding the equilibrium model) ranged between 0.001 and 0.003 per site, in line with the 243 observed values (Table 1 and 2). TD follows a U-shaped distribution for each $\mathrm{Nm}$ value as a 244 function of $T_{C O L}$, being more positive at recent $T_{C O L}$ and at equilibrium and less positive (or 245 negative for higher $\mathrm{Nm}$ ) at intermediate values. Therefore, species demography with $\mathrm{Nm} \sim 10$ 246 (and higher) and $T_{C O L}$ within $15 \mathrm{k}$ and $50 \mathrm{k}$ generations B.P. will have negative $T D$ values. In 247 contrast, species with lower $\mathrm{Nm}$ and very recent or very ancient $T_{C O L}$ will have positive $T D$. 248 This matches strikingly the $T D$ observed for the three shark species and their estimated 249 demographic parameters under SST (Table 1). We plot the normalized SFS and the stairwayplot 250 for all scenarios presented in Table 2 (Figures 4, 5, S1, S2 and S3). First, we note that none of 251 our scenarios, even those at equilibrium and with no variation in $\mathrm{Nm}$ through time, showed a 252 normalized SFS compatible with a constant size population (Figures 4, 5, S1, S2 and S3). The 253 normalized SFS and the reconstructed stairwayplot depend generally on the interaction between $254 N m$ and $T_{C O L}$ with a dynamic strikingly similar to $T D$ (which is indeed a summary of the SFS). 
255 For $N m=1$ we observed the signature of a recent decrease in $N e$ for all scenarios and 256 independently of $T_{C O L}$ (Figure 4). The normalized SFS showed consistently a strong deficit of

257 low frequency variants, typical of a demographic bottleneck and in agreement with the positive $258 T D$ (Figure 4 and Table 1). Furthermore, the stairwayplot could never detect the ancestral 259 expansion for any $T_{C O L}$. For growing $N m$, the interplay with $T_{C O L}$ becomes more complex. A 260 general result is that, once again, all scenarios were characterized by a recent decrease of $\mathrm{Ne}$ 261 when looking at the stairwayplot and a deficit of singletons compared to the other low 262 frequency classes when looking at the normalized SFS (Figures 5, S1 and S2). However, a 263 strong signature of ancestral expansion appeared for $N m>10$ and $T_{C O L}$ between $15 \mathrm{k}$ and $50 \mathrm{k}$ 264 generations B.P., mirroring the results of $T D$ for which most of these scenarios displayed a 265 negative value. Remarkably, the stairwayplot retrieved the ancestral expansion only slightly 266 overestimating the simulated $T_{C O L}$ (Figures 5, S1 and S2). Similar results were obtained for 267 FIM (Figures S4 and S5).

268 We compared SST vs SST-CH model (Figure 1) by means of the same ABC-RF model 269 selection framework previously adopted. The two models cannot be clearly distinguished in 270 any of the three structured species since: $i)$ they showed similar posterior probability $(\sim 0.50)$; 271 ii) the prior error rates are large $\sim 0.40$ (Table S9); iii) posterior distributions of $N m$ before and 272 after $T_{C H}$ are wide and largely overlapping (Table S10); $i v$ ) the normalized SFS closest to the 273 observed data retrieved under the two models are very similar (Figure S6). We ran a second set 274 of coalescent simulations focusing on the consequences of a recent change in connectivity on 275 the observed SFS and the reconstructed stairwayplot (Table S3 and S4). The decrease in 276 connectivity was simulated by reducing either $m$ (the migration rate per generation) or $N_{D E M E}$ 277 (the effective population size of each deme). As expected, we found a signature of recent 278 population decline in all simulated scenarios, with its intensity only slightly affected by the 
279 change in $N m$ (Figures 6, S7, S8 and S9). However, the drop in $N_{D E M E}$ (Figures 6 and S9) had 280 larger effect compared to the drop in $m$ (Figures S7 and S8) on both the normalized SFS and 281 the expansion time estimated by the stairwayplot. In scenarios with 100x reduction in $N_{D E M E}$, 282 the stairwayplot could not retrieve the ancestral expansion even for large long-term $\mathrm{Nm}$ (Figure 283 6). FIM-CH models displayed a behaviour similar to SST-CH models but more pronounced 284 (Figures S10, S11, S12 and S13, Table S5 and S6). While at $T_{C H}=10$ a decrease in $N m$ slightly 285 affected the SFS and the reconstructed stairwayplot, the consequence of the change in 286 connectivity are more substantial at $T_{C H}=50$, with a stronger deficit in singletons and a more 287 pronounced recent decline in $N e$ particularly in scenarios with a 100 -fold reduction of $N_{D E M E}$ 288 (Figures S11 and S13).

\section{Discussion}

Life history traits and demographic history of the four shark species

292 Discriminating whether the most appropriate model to reconstruct the demographic history of 293 a species is structured or unstructured should be the first step in empirical population genetics 294 investigations, particularly when targeting species of conservation concerns. Even when an 295 extensive spatial sampling is lacking, an ABC model selection approach can actually 296 distinguish whether the sampled deme belongs or not to a meta-population (similarly to 297 previous studies (Maisano Delser et al., 2019; Peter, Wegmann, \& Excoffier, 2010)). Among 298 the four species considered here, the tiger shark is the only panmictic. The three other species 299 conversely are best described by the SST model, i.e., the sampled populations belong to a meta300 population exchanging migrants following a stepping stone matrix. Our results reflect the tight 301 link between the level of meta-population structure (or its absence) and life history traits. The 
302 panmictic G. cuvier unsurprisingly can accomplish transoceanic movements and has the largest

303 body size among the sharks here considered (Table S1). In the three other species, the estimated

304 number of migrants $(\mathrm{Nm})$ remarkably follows the increase of movement range (Table 1 and S1)

305 and it is consistent with their behaviour and habitat use. Indeed, C. melanopterus, a strongly

306 lagoon dependent species, displays the lowest level of connectivity among the studied species

307 (Tables 1 and S1). These results bring meaningful hints about the influence of life history traits

308 on population structure in sharks, but more studies addressing this topic will be needed to

309 accurately detect which traits best predict its extent.

310 Gene genealogies in the four shark species and simulated scenarios

311 While it may seem counterintuitive to apply unstructured models to demes belonging to a meta-

312 population, we further investigated the demographic history of the four species by means of the

313 stairwayplot. When enough data is available, non-parametric unstructured models (such as the

314 PSMC (Li \& Durbin, 2011), the extended Bayesian skyline plot (Heled \& Drummond, 2008)

315 and the stairwayplot among others) provide a careful description of the distribution of 316 coalescence times of the gene genealogy, which ultimately depends from the "true"

317 demographic history (whether it is known or not) of the sampled lineages. If panmixia is the

318 most likely scenario, the distribution of coalescence times is directly related to the variation of

319 Ne through time and can therefore have a direct biological interpretation. This is the case for $G$.

320 cuvier (Table 1), whose reconstructed stairwayplot suggests that this species experienced a mild

321 ancestral expansion and a recent $\sim 4$-fold bottleneck around 2,000 years B.P. (consistent with

322 the results of Pirog et al. (2019), Figure 3). Conversely, signals detected by the stairwayplot in

323 the remaining three species, better described by the SST model (Table 1), cannot be directly

324 interpreted as changes in $\mathrm{Ne}$ over time. In this light, we ran coalescence simulations to provide 
325 helpful and general insights into the understanding of the relation between the inferences 326 performed under unstructured and structured models.

327 We first focus on scenarios simulated under the SST, with parameters close to those estimated 328 in real data. The first and most striking result is that we systematically observed a recent 329 bottleneck under all simulated scenarios (Table 2, Figures 4, 5, S1, S2 and S3). This result could 330 seem at a first glance surprising and due to an artefact. However, this is not the case, as: i) the 331 signal does not depend on the inferential algorithm chosen to analyse the data (i.e., the 332 stairwayplot), since the normalized spectra showed a deficit in singletons compared to the other 333 low frequency classes (Figures 4, 5, S1 and S2), which is typical of a recent population decline; ii) it is consistent with the distribution of the Inverse Instantaneous Coalescence Rate (IICR) computed in one diploid individual, which shows a signature of decline under similar metapopulation models (Chikhi et al., 2018; Mazet, Rodríguez, Grusea, Boitard, \& Chikhi, 2016;

337 Rodríguez et al., 2018). The results of our simulations are consistent with the recent bottleneck 338 observed in the three shark species (Figure 3), with its intensity inversely correlated to the estimated Nm (i.e., stronger for C. melanopterus and C. limbatus than for C. amblyrhynchos).

340 In our SST model there is an instantaneous colonization of the array of demes at $T_{C O L}$, which 341 corresponds also to a demographic expansion (i.e., the total number of individuals in the array 342 of deme is larger than those in the ancestral deme). However, this signature is detected only for $343 N m \geq 5$ when $T_{C O L}$ is neither too recent nor too old (at equilibrium) (Figures 5, S1, S2 and S3).

344 In these scenarios, the beginning of the expansion retrieved by the stairwayplot broadly 345 corresponds to the simulated $T_{C O L}$. This again corroborates the results obtained for the three 346 shark species, since the two species with higher $N m$ displayed indeed an ancestral expansion in 347 the stairwayplot with a timing consistent with the estimated $T_{C O L}$ (Table 1, Figures 2 and 3). 348 Similarly, it explains why we could not retrieve the ancestral expansion for C. melanopterus 
349 nor estimate $T_{C O L}$ under the SST model: this appears to be a property of the coalescence pattern

350 and it is not related to the amount of data available (see below).

Coalescence phases in structured models

352 It is now straightforward to frame all these findings under the coalescence perspective. The 353 coalescence history of the lineages sampled from a single deme in an SST (or FIM) model can

354 be separated for simplicity into three phases: the scattering, the collecting and the ancestral 355 phase (Figure 7). Going backward in time, lineages will coalesce in the sampled deme with a 356 rate according to both $N m$ and $N_{D E M E}$ until all lineages either have coalesced or migrated to 357 another deme. This is the scattering phase described in the seminal works of (Wakeley, 1998, 358 1999). The scattering phase was considered instantaneous for mathematical tractability, with 359 its outcome dependent on $\mathrm{Nm}$ only, but later works could disentangle the effect of $N_{D E M E}$ and $360 m$ on the shape of the gene genealogy (Mona, 2017). The collecting phase starts when the 361 lineages which did not coalesce have migrated to other demes of the array: they will then

362 coalesce according to a Kingman process with a rate scaled by $\mathrm{Nm}$ and the number of demes $d$ 363 of the array (Wakeley, 1999) (Figure 7). Finally, all surviving lineages (in non-equilibrium

364 model) will reach the ancestral deme at $T_{C O L}$, where they will coalesce at a rate depending only 365 on the $N_{A N C}$ parameter (Figure 7). The interplay between the demographic parameters $\left(N_{D E M E}\right.$, $\left.366 N m, N_{A N C}, d\right)$ and the historical events $\left(T_{C O L}\right.$ and $\left.T_{C H}\right)$ determines the length of each coalescence 367 phase and the resulting shape of the gene genealogy of the sampled lineages (Figure 7).

368 In species with low $N m$, the rate of coalescence during the scattering phase is very fast since 369 lineages have low probability of emigrating from the sampled deme and high probability of 370 coalescence due to the small $N$. Once all the lineages are dispersed in the array of demes, there 371 will be two possible outcomes: i) in equilibrium model, we shift to the collecting phase, where 372 the rate of coalescence drops since lineages will hardly fall in the same deme again; ii) in non- 
373 equilibrium model, with the parameters we have simulated here, there will be very few (if any) 374 coalescence events during the collecting phase and the transition will be directly from the 375 scattering to the ancestral phase. Both the collecting and the ancestral phases have a rate of 376 coalescence lower than the scattering phase, which determines the observed recent drop in $\mathrm{Ne}$ 377 for all simulated scenarios. Remarkably, the decline in $\mathrm{Ne}$ is much stronger in equilibrium 378 model, since the rate of coalescence is much lower in the collecting than in the ancestral phase 379 (Figures 4, 5, S1, S2 and S3). Low Nm species will therefore have only two coalescence phases, 380 the scattering and either the collecting (in equilibrium model) or the ancestral (in non381 equilibrium model) which is why the signature of the ancestral expansion is lost.

382 For growing $\mathrm{Nm}$, in equilibrium model there will be again only two coalescence phases, namely 383 the scattering and collecting, with the latter having a lower rate of coalescence than the former 384 independently of the simulated parameters. This is why we observed always a strong bottleneck 385 consistent with the distribution of the IICR statistics in any equilibrium model (Chikhi et al., 386 2018; Mazet et al., 2015; Rodríguez et al., 2018). In non-equilibrium model, there will be two 387 different situations: a) $T_{C O L}$ (in generations) is of the same order of the deme size $N_{D E M E}$. In this 388 setting, going backward in time few lineages would have escaped the sampled demes before $389 T_{C O L}$. This corresponds to a shift in the coalescence rate directly from the scattering to the 390 ancestral phase, resulting in a bottleneck of lower intensity compared to an equilibrium model 391 (Figures 4, 5, S1 and S2), for the same reasons as above; b) $T_{C O L}$ (in generations) is larger than $392 N_{D E M E}$. In this setting, some coalescence events may occur during the collecting phase, at a rate 393 much slower than the two other phases. This determines the hump observed in the stairwayplot

394 (Figures 4, 5, S1 and S2) and explains why in this window of parameters it is also possible to 395 correctly estimate $T_{C O L}$ using our ABC framework. Further simulations under the FIM model 396 confirmed those patterns even though the ancestral expansion could be detected for lower long- 
397 term $\mathrm{Nm}$ than the corresponding SST scenario (Figure S4). This is probably due to a higher

398 apparent connectivity underlined the by FIM, where lineages can move more freely during the

399 collecting phase in comparison to SST where migrants only come from the closest neighbours.

400 If many coalescence events occur during the collecting phase, the change in coalescence rate

401 will affect the resulting gene genealogy and it will be detected by the stairwayplot (or any other

402 unstructured method based on coalescent theory).

403 Changes in connectivity

404 Using coalescence arguments, we clarified why simple meta-population models with constant 405 connectivity generate a gene genealogy harbouring a signature of a recent decline for any 406 parameters' combination. The signature of bottleneck detected by the stairwayplot in the three 407 shark species best described by SST can be therefore interpreted as a consequence of the 408 underlying structure. However, connectivity likely changes through time. For instance, human 409 activities have likely impacted the evolutionary history of a large number of species either by 410 decreasing their effective population size and/or by fragmenting their habitat (i.e., reducing 411 migration rates between demes). This intuitively should exacerbate the signature of population 412 decline in the resulting gene genealogy. However, it remains to be shown whether this signature 413 is qualitatively and quantitatively distinguishable from models with constant connectivity. This 414 is a question of fundamental importance to understand whether it is possible to detect recent 415 bottleneck in structured populations. To this end, we further investigated by coalescent 416 simulations the expected gene genealogy in SST-CH (and FIM-CH) models with a change in 417 connectivity 10 or 50 generations B.P., which matches the beginning of extensive 418 anthropogenic influence on biodiversity considering our species' generation time (Ceballos et 419 al., 2015). The resulting gene genealogies were poorly affected by the recent drop in 420 connectivity, with both the normalized SFS and the inferred $N e$ dynamic following the same 
421 trajectory of the corresponding scenario with the same long-term $\mathrm{Nm}$ and $T_{C O L}$ (Figures 6, S7, $422 \mathrm{~S} 8, \mathrm{~S} 9, \mathrm{~S} 10, \mathrm{~S} 11, \mathrm{~S} 12$ and S13). We noticed the drop in $N_{D E M E}$ (Figures 6, S9, S12 and S13) 423 had stronger influence than the drop in $m$ (Figures S7, S8, S10 and S11), consistent with 424 previous finding showing that the distribution of coalescence events depends not only by the $425 N m$ compound parameter but also by their individuals values (Mona, 2017). This can be 426 explained once again in the light of the length of the coalescence phases (Figure 7). Reducing $427 N_{D E M E}$ will increase exponentially the number of coalescence events, drastically shortening the 428 scattering phase and the number of surviving lineages. Reducing $m$ will only linearly reduce 429 the probability of migrations outside the deme, marginally affecting the length of the scattering 430 phase and the number of surviving lineages compared to constant $N m$ scenarios. This is why a 431 100-fold reduction in $N_{D E M E}$ significantly reduces the number of lineages entering in the 432 collecting phase, almost hiding the ancestral expansion in high long-term $\mathrm{Nm}$ scenarios (Figures 433 6, S9, S12 and S13), while a 100-fold reduction in $m$ is barely detectable (Figures S7, S8, S10 434 and S11). Similarly, the recent reduction in either $N_{D E M E}$ or $m$ cannot be detected for lower 435 long-term $\mathrm{Nm}$ scenarios, where the collecting phase is already missing. This explains why the 436 general pattern is strikingly similar between SST-CH and SST simulations, which implies that 437 the simulated change in connectivity is too recent to significantly alter the pattern of 438 coalescence events and that a recent drop can be hardly detected on the basis of the SFS only. 439 Our empirical data are consistent with these findings: when we compared SST vs. SST-CH 440 models in the three shark species using the $\mathrm{ABC}$ framework, we failed to clearly distinguish 441 the two models (Tables S9 and S10, Figure S6). This seems to be a paradox: we observed a 442 recent bottleneck in species of conservation concern using unstructured model, but we cannot 443 exclude that this is just the consequence of population structure.

$444 \quad$ Practical recommendations and conservation concerns 
445 This study highlight once more the importance to explicitly test for meta-population structure

446 before interpreting the demographic signals detected by unstructured models, similarly to what

447 advocated previously by (Maisano Delser et al., 2019; Rodríguez et al., 2018). If the meta-

448 population structure hypothesis is rejected, the variation of $N e$ through time can be directly

449 interpreted as the demographic history of the population under investigation, such as the case

450 of tiger shark. Otherwise, this variation is still related to demographic events, but it has to be

451 explained in the light of population structure and its consequence on the rate of coalescence

452 events. We showed by coalescent simulations how to interpret such variation: the recent

453 bottleneck detected by the stairwayplot in demes belonging to a meta-population is a

454 consequence of the coalescence process. In other words, any inferential method implementing

455 an unstructured model will detect such decline (if enough data is available) since it is a property

456 of the gene genealogy. Importantly, the gene genealogy is only slightly affected by recent

457 changes in connectivity if the time of this change in generations is of the same order of the size

458 of the deme.

459 Our study underscore a key issue in conservation genetics as a recent decline inferred by an

460 unstructured model can be mis-interpreted as a consequence of recent anthropic pressures

461 (Ceballos et al., 2015) when it actually results from meta-population structure. This is all the

462 more alarming since the majority of species is likely organised in meta-populations across their

463 range rather than panmictic at a large scale. We therefore stress the necessity for an educated

464 choice of tools to correctly uncover the recent trend of a species and design proper conservation

465 programs. For instance, detecting a recent bottleneck in meta-populations will require summary

466 statistics measuring the linkage disequilibrium (Boitard, Rodríguez, Jay, Mona, \& Austerlitz,

467 2016; Kerdoncuff, Lambert, \& Achaz, 2020) and/or the inferential framework based on the

468 IICR (Chikhi et al., 2018; Rodríguez et al., 2018) coupled with whole genome data. On a 
469 positive note, we showed that the colonization time of the array of demes can be estimated to 470 some extent (and under some combinations of parameters) by unstructured models. We believe 471 that this is particularly important because it has been shown that the simple instantaneous 472 colonization process we used here behaves similarly to a spatial explicit range expansion 473 (Hamilton, Stoneking, \& Excoffier, 2005; Mona, 2017), which is certainly a more realistic 474 model but more difficult to investigate. We are aware that the meta-population models here 475 tested are simple and the parameters chosen are specific of the three shark species we focused 476 on. Nevertheless, the time-scale separation of the coalescence process is general, and it allows 477 explaining intuitively any structured models. The four shark species here used as an example 478 has the merit to cover a large spectrum of LHT and consequently a large spectrum of 479 demographic scenarios, going from a highly structured to a panmictic population: this has 480 strong implications on the distribution of coalescence times and therefore on the interpretation 481 of the observed data.

\section{Conclusion}

483 In this study we found that population structure, independently from the degree of connectivity 484 between demes and the migration matrix relating them, intrinsically determines a variation in 485 the rate of coalescence events through time. We showed that the intensity and the direction(s) 486 of such variation related to the demographic parameters of the meta-population in a predictable 487 way. Our results highlight the importance of detecting population structure (which depends on 488 LHT among other factors) before performing any demographic inferences but, at the same time, 489 they reveal the utility of unstructured models to describe the shape of the gene genealogy, which 490 is the final product of the evolutionary history of a species. A combination of structured and 491 unstructured models (better if non-parametric) is therefore the key to best characterize the 492 evolutionary history of a species. We call for a change in perspective when investigating the 
493

494

495

496

497

498

499

500

501

502

503

504

505

506

507

508

509

510

511

512

513

514

515

516

517

518

519

520

521

522

523

524

525

526

527

demographic history of a species: the focus should be put in the reconstruction of the variation of both $N$ and $m$ through time, which requires certainly new methodological development and probably more data.

\section{Acknowledgement}

We are grateful to the Genotoul bioinformatics platform Toulouse Midi-Pyrenees (Bioinfo Genotoul; http://bioinfo.genotoul.fr/) for providing computing resources. We are indebted to Oscar Lao for fruitful discussions and careful reading of the manuscript. This work was supported by two ATM grants (2016 and 2017) from the Muséum National d'Histoire Naturelle to S.M.

\section{References}

Arenas, M., Ray, N., Currat, M., \& Excoffier, L. (2012). Consequences of range contractions and range shifts on molecular diversity. Molecular Biology and Evolution, 29(1), 207218. https://doi.org/10.1093/molbev/msr187

Bertorelle, G., Benazzo, A., \& Mona, S. (2010). ABC as a flexible framework to estimate demography over space and time: Some cons, many pros. Molecular Ecology, 19(13), 2609-2625. https://doi.org/10.1111/j.1365-294X.2010.04690.x

Boitard, S., Rodríguez, W., Jay, F., Mona, S., \& Austerlitz, F. (2016). Inferring Population Size History from Large Samples of Genome-Wide Molecular Data - An Approximate Bayesian Computation Approach. PLoS Genetics, 12(3), e1005877. https://doi.org/10.1371/journal.pgen.1005877

Ceballos, G., Ehrlich, P. R., Barnosky, A. D., García, A., Pringle, R. M., \& Palmer, T. M. (2015). Accelerated modern human-induced species losses: Entering the sixth mass extinction. Science Advances, 1(5), 9-13. https://doi.org/10.1126/sciadv.1400253

Chikhi, L., Rodríguez, W., Grusea, S., Santos, P., Boitard, S., \& Mazet, O. (2018). The IICR (inverse instantaneous coalescence rate) as a summary of genomic diversity: Insights into demographic inference and model choice. Heredity, 120(1), 13-24. https://doi.org/10.1038/s41437-017-0005-6

Chikhi, L., Sousa, V. C., Luisi, P., Goossens, B., \& Beaumont, M. A. (2010). The Confounding Effects of Population Structure, Genetic Diversity and the Sampling Scheme on the Detection and Quantification of Population Size Changes. Genetics, 186(3), 983-995. https://doi.org/10.1534/genetics.110.118661

Corrigan, S., Lowther, A. D., Beheregaray, L. B., Bruce, B. D., Cliff, G., Duffy, C. A., ... 
Rogers, P. J. (2018). Population Connectivity of the Highly Migratory Shortfin Mako (Isurus oxyrinchus Rafinesque 1810) and Implications for Management in the Southern Hemisphere. Frontiers in Ecology and Evolution, 6(NOV), 1-15. https://doi.org/10.3389/fevo.2018.00187

Cortés, E. (2002). Incorporating uncertainty into demographic modeling: Application to shark populations and their conservation. Conservation Biology, 16(4), 1048-1062. https://doi.org/10.1046/j.1523-1739.2002.00423.x

Excoffier, L., Dupanloup, I., Huerta-Sánchez, E., Sousa, V. C., \& Foll, M. (2013). Robust Demographic Inference from Genomic and SNP Data. PLoS Genetics, 9(10), e1003905. https://doi.org/10.1371/journal.pgen.1003905

Excoffier, L., Foll, M., \& Petit, R. J. (2009). Genetic Consequences of Range Expansions. Annual Review of Ecology, Evolution, and Systematics, 40(1), 481-501. https://doi.org/10.1146/annurev.ecolsys.39.110707.173414

Hamilton, G., Stoneking, M., \& Excoffier, L. (2005). Molecular analysis reveals tighter social regulation of immigration in patrilocal populations than in matrilocal populations. Proceedings of the National Academy of Sciences of the United States of America, 102(21), 7476-7480. https://doi.org/10.1073/pnas.0409253102

Heled, J., \& Drummond, A. J. (2008). Bayesian inference of population size history from multiple loci. BMC Evolutionary Biology, 8(1), 289. https://doi.org/10.1186/1471-21488-289

Heller, R., Nursyifa, C., Garcia-Erill, G., Salmona, J., Chikhi, L., Meisner, J., ... Albrechtsen, A. (2021). A reference-free approach to analyse RADseq data using standard next generation sequencing toolkits. Molecular Ecology Resources, 21(4), 1085-1097. https://doi.org/10.1111/1755-0998.13324

Karl, S. A., Motta, P. J., Stewart, B. S., Wilson, S. G., Bowen, B. W., Castro, A. L. F., ... Hueter, R. E. (2010). Population genetic structure of Earth's largest fish, the whale shark (Rhincodon typus). Molecular Ecology, 16(24), 5183-5192. https://doi.org/10.1111/j.1365-294x.2007.03597.x

Kerdoncuff, E., Lambert, A., \& Achaz, G. (2020). Testing for population decline using maximal linkage disequilibrium blocks. Theoretical Population Biology, 134, 171-181. https://doi.org/10.1016/j.tpb.2020.03.004

Khimoun, A., Doums, C., Molet, M., Kaufmann, B., Peronnet, R., Eyer, P. A., \& Mona, S. (2020). Urbanization without isolation: The absence of genetic structure among cities and forests in the tiny acorn ant Temnothorax nylanderi. Biology Letters, 16(1). https://doi.org/10.1098/rsbl.2019.0741

Korneliussen, T. S., Albrechtsen, A., \& Nielsen, R. (2014). ANGSD: Analysis of Next Generation Sequencing Data. BMC Bioinformatics, 15(1), 1-13. https://doi.org/10.1186/s12859-014-0356-4

Lapierre, M., Lambert, A., \& Achaz, G. (2017). Accuracy of Demographic Inferences from the Site Frequency Spectrum: The Case of the Yoruba Population. Genetics, 206(1), 439-449. https://doi.org/10.1534/genetics.116.192708

Li, H., \& Durbin, R. (2009). Fast and accurate short read alignment with Burrows-Wheeler transform. Bioinformatics, 25(14), 1754-1760. https://doi.org/10.1093/bioinformatics/btp324

Li, H., \& Durbin, R. (2011). Inference of human population history from individual wholegenome sequences. Nature, 475(7357), 493-496. https://doi.org/10.1038/nature10231

Liu, X., \& Fu, Y.-X. (2015). Exploring population size changes using SNP frequency spectra. Nature Genetics, 47(5), 555-559. https://doi.org/10.1038/ng.3254 
Maisano Delser, P., Corrigan, S., Duckett, D., Suwalski, A., Veuille, M., Planes, S., ... Mona, S. (2019). Demographic inferences after a range expansion can be biased: the test case of the blacktip reef shark (Carcharhinus melanopterus). Heredity, 122(6), 759-769. https://doi.org/10.1038/s41437-018-0164-0

Maisano Delser, P., Corrigan, S., Hale, M., Li, C., Veuille, M., Planes, S., ... Mona, S. (2016). Population genomics of $C$. melanopterus using target gene capture data: Demographic inferences and conservation perspectives. Scientific Reports, 6(April), 112. https://doi.org/10.1038/srep33753

Mazet, O., Rodríguez, W., Grusea, S., Boitard, S., \& Chikhi, L. (2016). On the importance of being structured: instantaneous coalescence rates and human evolution-lessons for ancestral population size inference? Heredity, 116(4), 362-371. https://doi.org/10.1038/hdy.2015.104

Mazet, Olivier, Rodríguez, W., \& Chikhi, L. (2015). Demographic inference using genetic data from a single individual: Separating population size variation from population structure. Theoretical Population Biology, 104, 46-58. https://doi.org/10.1016/j.tpb.2015.06.003

Mona, S. (2017). On the role played by the carrying capacity and the ancestral population size during a range expansion. Heredity, 118(2), 143-153. https://doi.org/10.1038/hdy.2016.73

Mona, S., Ray, N., Arenas, M., \& Excoffier, L. (2014). Genetic consequences of habitat fragmentation during a range expansion. Heredity, 112(3), 291-299. https://doi.org/10.1038/hdy.2013.105

Paris, J. R., Stevens, J. R., \& Catchen, J. M. (2017). Lost in parameter space: a road map for stacks. Methods in Ecology and Evolution, 8(10), 1360-1373. https://doi.org/10.1111/2041-210X.12775

Parsons, G. R. (1990). Metabolism and swimming efficiency of the bonnethead shark Sphyrna tiburo. Marine Biology, 104(3), 363-367. https://doi.org/10.1007/BF01314338

Peter, B. M., Wegmann, D., \& Excoffier, L. (2010). Distinguishing between population bottleneck and population subdivision by a Bayesian model choice procedure. Molecular Ecology, 19(21), 4648-4660. https://doi.org/10.1111/j.1365-294X.2010.04783.x

Peterson, B. K., Weber, J. N., Kay, E. H., Fisher, H. S., \& Hoekstra, H. E. (2012). Double Digest RADseq: An Inexpensive Method for De Novo SNP Discovery and Genotyping in Model and Non-Model Species. PLoS ONE, 7(5), e37135. https://doi.org/10.1371/journal.pone.0037135

Pirog, A., Jaquemet, S., Ravigné, V., Cliff, G., Clua, E., Holmes, B. J., ... Magalon, H. (2019). Genetic population structure and demography of an apex predator, the tiger shark Galeocerdo cuvier. Ecology and Evolution, 9(10), 5551-5571. https://doi.org/10.1002/ece3.5111

Pudlo, P., Marin, J.-M. M., Estoup, A., Cornuet, J.-M. M., Gautier, M., \& Robert, C. P. (2016). Reliable ABC model choice via random forests. Bioinformatics, 32(6), 859-866. https://doi.org/10.1093/bioinformatics/btv684

Ray, N., Currat, M., \& Excoffier, L. (2003). Intra-deme molecular diversity in spatially expanding populations. Molecular Biology and Evolution, 20(1), 76-86. https://doi.org/10.1093/molbev/msg009

Raynal, L., Marin, J. M., Pudlo, P., Ribatet, M., Robert, C. P., \& Estoup, A. (2019). ABC random forests for Bayesian parameter inference. Bioinformatics, 35(10), 1720-1728. https://doi.org/10.1093/bioinformatics/bty867

Rochette, N. C., Rivera-Colón, A. G., \& Catchen, J. M. (2019). Stacks 2: Analytical methods 
624 for paired-end sequencing improve RADseq-based population genomics. Molecular $625 \quad$ Ecology, 28(21), 4737-4754. https://doi.org/10.1111/mec.15253

626

627

628

629

630

631

632

633

634

635

636

637

638

639

640

641

642

643

644

645

646

647

648

649

650

651

652

653

654
Rodríguez, W., Mazet, O., Grusea, S., Arredondo, A., Corujo, J. M., Boitard, S., \& Chikhi, L. (2018). The IICR and the non-stationary structured coalescent: towards demographic inference with arbitrary changes in population structure. Heredity, 121(6), 663-678. https://doi.org/10.1038/s41437-018-0148-0

Städler, T., Haubold, B., Merino, C., Stephan, W., \& Pfaffelhuber, P. (2009). The impact of sampling schemes on the site frequency spectrum in nonequilibrium subdivided populations. Genetics, 182(1), 205-216. https://doi.org/10.1534/genetics.108.094904

Tajima, F. (1989). Statistical method for testing the neutral mutation hypothesis by DNA polymorphism. Genetics, 123(3), 585-595. https://doi.org/10.1093/genetics/123.3.585

Wakeley, J. (1998). Segregating Sites in Wright's Island Model. Theoretical Population Biology, 53, 166-174.

Wakeley, J. (1999). Nonequilibrium migration in human history. Genetics, 153(4), 18631871.

Warmuth, V. M., \& Ellegren, H. (2019). Genotype-free estimation of allele frequencies reduces bias and improves demographic inference from RADSeq data. Molecular Ecology Resources, 19(3), 586-596. https://doi.org/10.1111/1755-0998.12990

Watterson, G. A. A. (1975). On the number of segregating sites in genetical models without recombination. Theoretical Population Biology, 7(2), 256-276. https://doi.org/10.1016/0040-5809(75)90020-9

\section{Data availability statement}

Fastq sequence files, SFS and scripts are available from the Dryad Digital Repository:

https://doi.org/10.5061/dryad.b8gtht7d1.

\section{Authors contribution}

S.M. and P.L. conceived the project. S.P. provided reagents and samples. S.M. and P.L analysed the data and wrote the manuscript with input from S.P. 


\section{Tables}

Table 1. Summary statistics and ABC estimation. Number of loci and SNPs after filtering, mean pairwise difference $\left(\theta_{\pi}\right)$, Watterson theta $\left(\theta_{\mathrm{w}}\right)$, Tajima's $D(T D)$, posterior probability of the most supported model and its parameters (median value and $95 \%$ credible interval in parentheses).

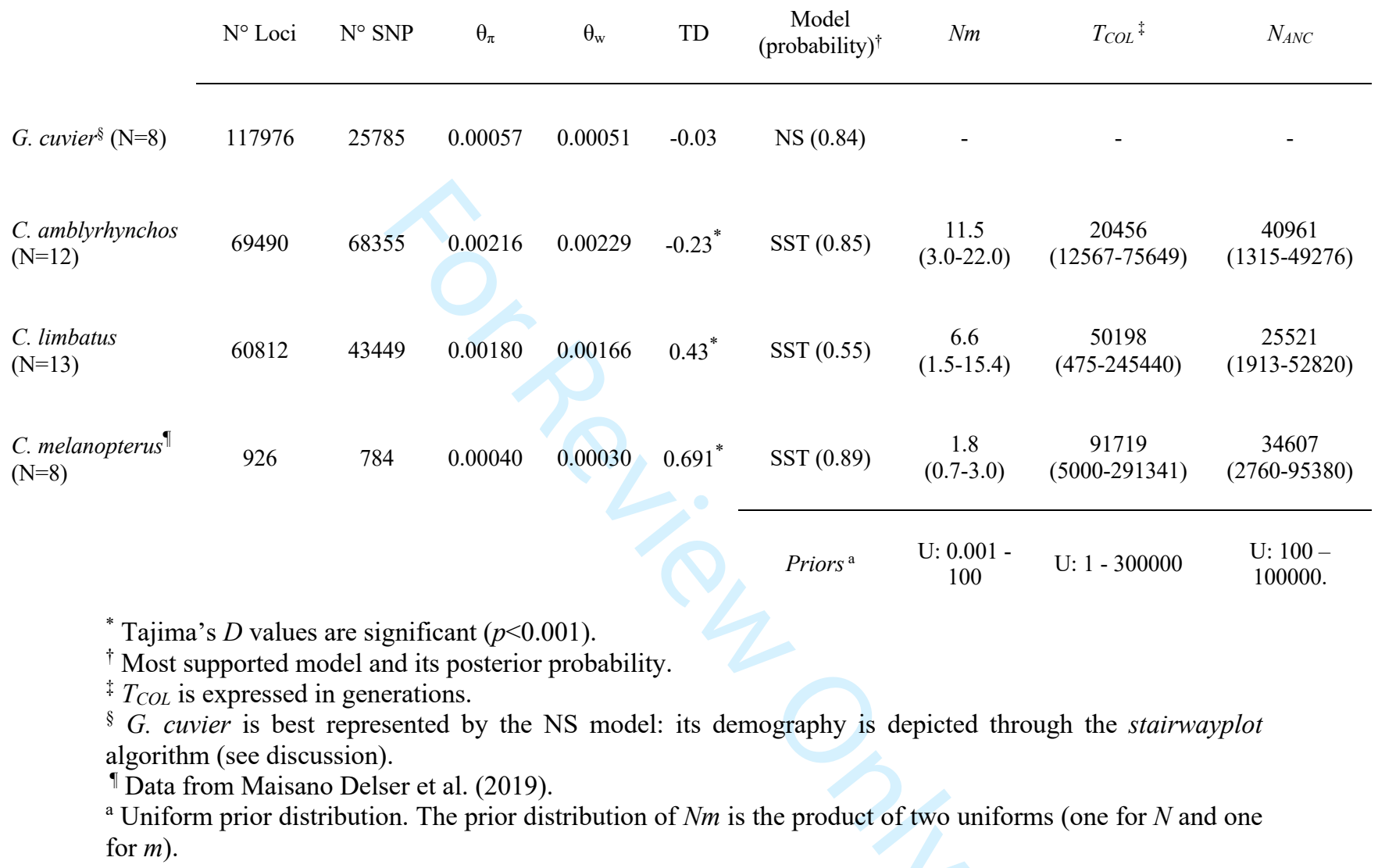


Table 2. Coalescent simulations of 50,000 Rad-loci under SST model, with mutation rate fixed to $1.93 * 10^{-8}$ per site per generation and $N_{A N C}$ fixed to 50,000 . Mean pairwise difference $\left(\theta_{\pi}\right)$, Watterson theta $\left(\theta_{\mathrm{w}}\right)$, Tajima's D (TD), and number of segregating sites (S) are averaged over 100 replicates.

\begin{tabular}{cccccc}
$\boldsymbol{N m}$ & $\boldsymbol{T}_{\boldsymbol{C O L}}$ & $\boldsymbol{\theta}_{\boldsymbol{\pi}}{ }^{\ddagger}$ & $\boldsymbol{\theta}_{\mathbf{s}}{ }^{*}$ & TD & $\mathbf{S}$ \\
\hline 1 & 5000 & 0.0013 & 0.0011 & 0.531 & 23599 \\
& 15000 & 0.0013 & 0.0012 & 0.405 & 24094 \\
& 50000 & 0.0017 & 0.0016 & 0.406 & 32201 \\
& $\infty^{\dagger}$ & 0.0161 & 0.0139 & 0.669 & 283564 \\
\hline 5 & 5000 & 0.0017 & 0.0016 & 0.361 & 32443 \\
& 15000 & 0.0019 & 0.0018 & 0.191 & 37712 \\
& 50000 & 0.0028 & 0.0028 & 0.035 & 56474 \\
& $\infty$ & 0.0177 & 0.0150 & 0.749 & 306786 \\
\hline \multirow{2}{*}{10} & 5000 & 0.0019 & 0.0018 & 0.180 & 36561 \\
& 15000 & 0.0021 & 0.0022 & -0.087 & 44380 \\
& 50000 & 0.0031 & 0.0034 & -0.364 & 69436 \\
& $\infty$ & 0.0180 & 0.0158 & 0.585 & 321619 \\
\hline & 5000 & 0.0019 & 0.0019 & 0.048 & 38919 \\
& 15000 & 0.0022 & 0.0024 & -0.274 & 48479 \\
& 50000 & 0.0032 & 0.0038 & -0.608 & 77391 \\
& $\infty$ & 0.0181 & 0.0163 & 0.465 & 331816 \\
\hline
\end{tabular}

\footnotetext{
${ }^{\dagger}$ Equilibrium model obtained by simulating $T_{C O L}=\infty$.

\$ Theta values are expressed per site per generation.
} 


\section{Figure Legends}

Figure 1. Evolutionary scenarios considered in this study (to both infer parameters in real data under an $\mathrm{ABC}$ framework and to perform coalescent simulations). SST (FIM) model is a simplified version of SST-CH (FIM-CH) in which connectivity $N m$ is constant after $T_{C O L}$. Details on each parameter are presented in the main text.

Figure 2. Posterior distribution of the number of migrants per generation $\mathrm{Nm}$ (panel A) and of the colonisation time of the array of deme $T_{C O L}$ (panel B) estimated under the stepping stone model (SST) for Carcharhinus amblyrhynchos (red), Carcharhinus limbatus (green) and Carcharhinus melanopterus (blue).

Figure 3. Panel A: variation of the effective population size $(\mathrm{Ne})$ through time and its $75 \%$ confidence interval estimated by the stairwayplot. Panel B: normalized SFS computed as in (Lapierre et al., 2017). Carcharhinus amblyrhynchos is represented in red, Carcharhinus limbatus in green, Carcharhinus melanopterus in blue, and Galeocerdo cuvier in purple.

Figure 4. stairwayplot (maximum likelihood $\mathrm{Ne}$ and $75 \%$ confidence interval) (panel A) and normalized SFS (panel B) computed in simulated non-equilibrium SST scenarios with $N m=1$, averaged over 100 replicates. Colonisation time of the array of deme $T_{C O L}$ occurred 5,000 (red), 15,000 (blue), and 50,000 (green) generations B.P., visually represented by the vertical dashed lines in panel A. The normalized SFS expected under a constant size non-structured model (NS constant size) is also shown (grey dashed line in panel B).

Figure 5. stairwayplot (maximum likelihood $\mathrm{Ne}$ and $75 \%$ confidence interval) (panel A) and normalized SFS (panel B) computed in simulated non-equilibrium SST scenarios with $N m=10$, averaged over 100 replicates. Colonisation time of the array of deme $T_{C O L}$ occurred 5,000 (red), 15,000 (blue), and 50,000 (green) generations B.P., visually represented by the vertical dashed lines in panel A. The normalized SFS expected under a constant size non-structured model (NS constant size) is also shown (grey dashed line in panel B).

Figure 6. stairwayplot (maximum likelihood $\mathrm{Ne}$ ) (panel A) and normalized SFS (panel B) computed in simulated non-equilibrium SST scenarios with $T_{C O L}=15,000$ generations B.P. and an instantaneous decrease of the deme size $\left(N_{D E M E}\right)$ forward in time at $T_{C H}=10$ generations B.P. Colours represent the long-term connectivity values: $N m=1$ (blue), $N m=5$ (green), $N m=10$ (red), $N m=15$ (black). Line style represents the 10-fold (small dashes) or 100-fold (dots) reduction of $N_{D E M E}$, or constant $\mathrm{Nm}$ (continuous line). The vertical grey dashed line in panel A represents the simulated colonisation time $T_{C O L}$.

Figure 7. Schematic diagram representing the different coalescence phases in the history of lineages sampled from a deme belonging to a non-equilibrium meta-population. Each phase and related parameters are represented by a colour. Parameters influencing the coalescence rate in each phase are: the effective size of the deme $\left(N_{D E M E}\right)$ and the migration rate $(m)$ for the scattering phase; the number of migrants exchanged per generation $(\mathrm{Nm})$ and the number of demes $(d)$ for the collecting phase; and the ancestral effective size $\left(N_{A N C}\right)$ for the ancestral phase. 


\section{Figures}

Model NS

Model FIM-CH

Model SST-CH
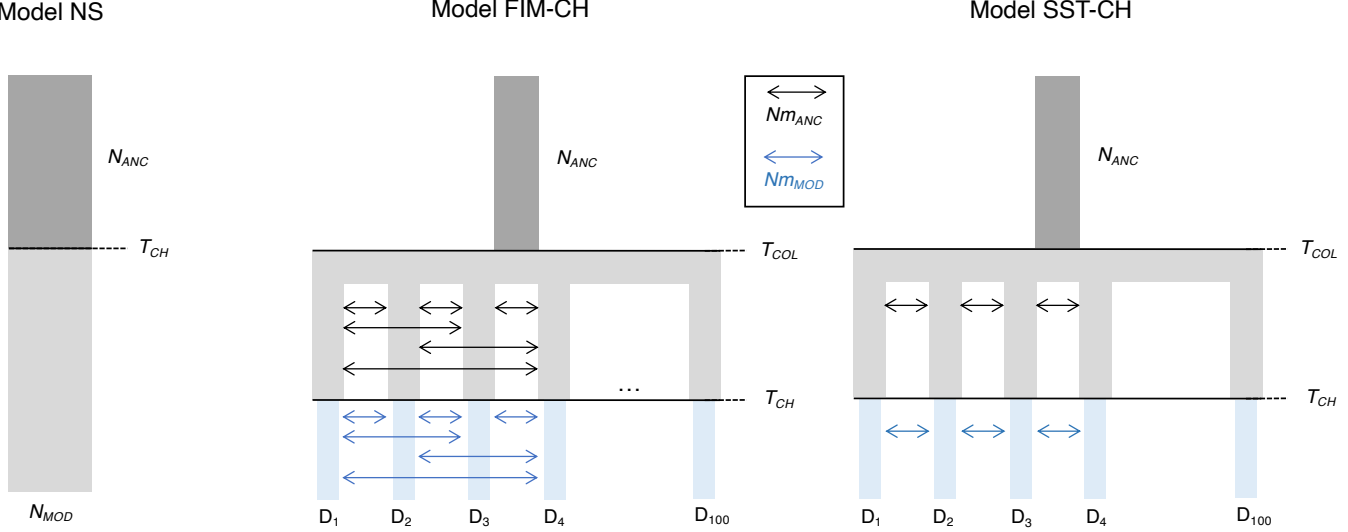

656 

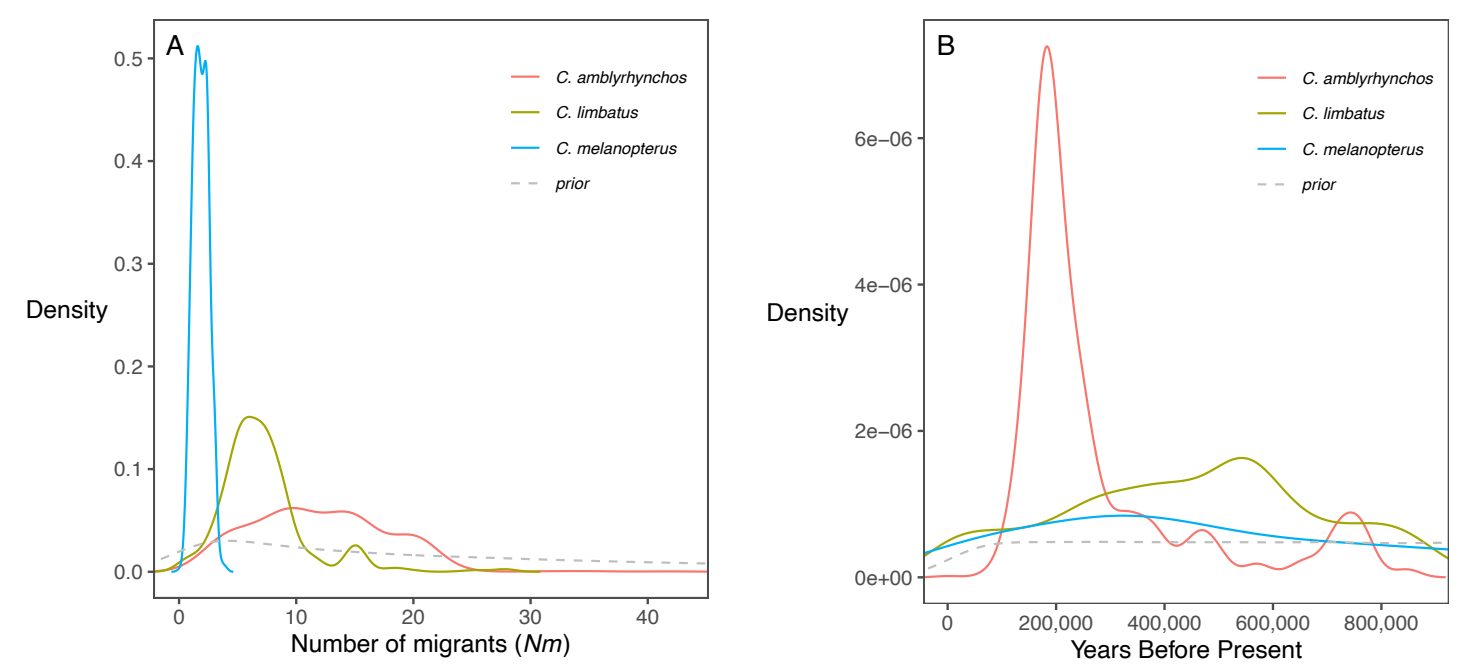

657 

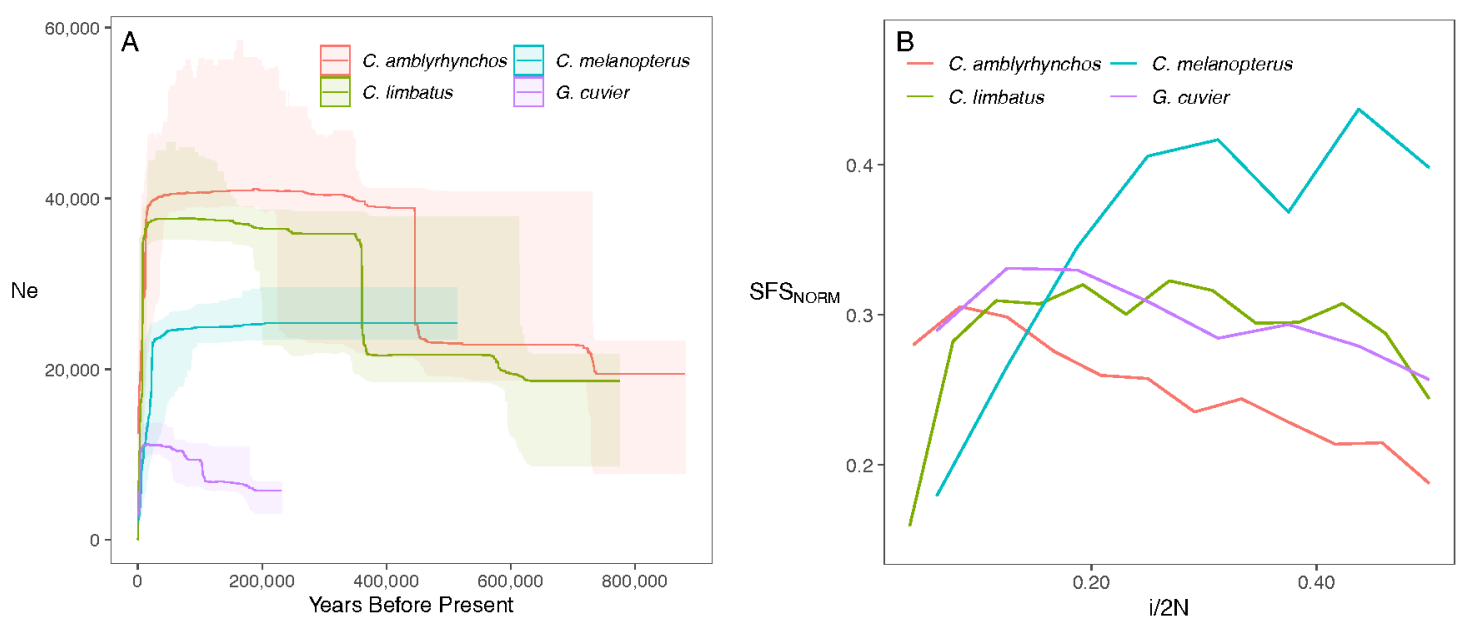

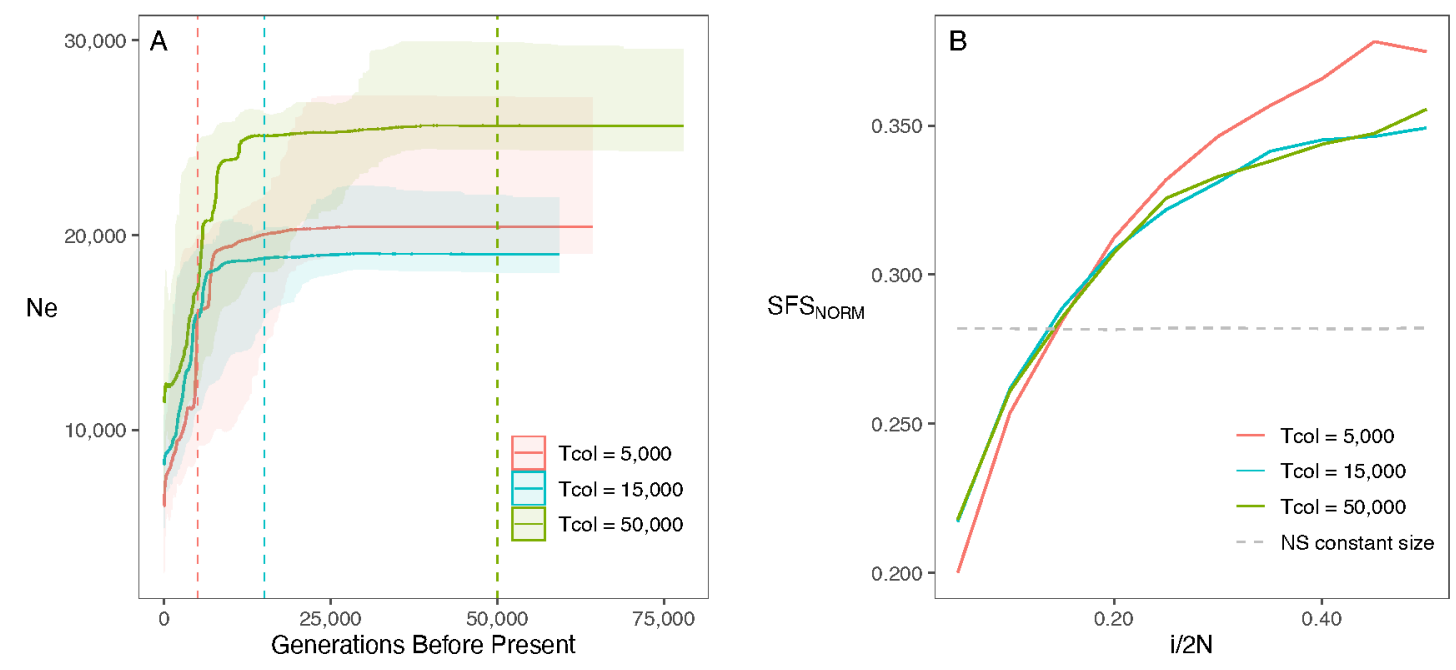

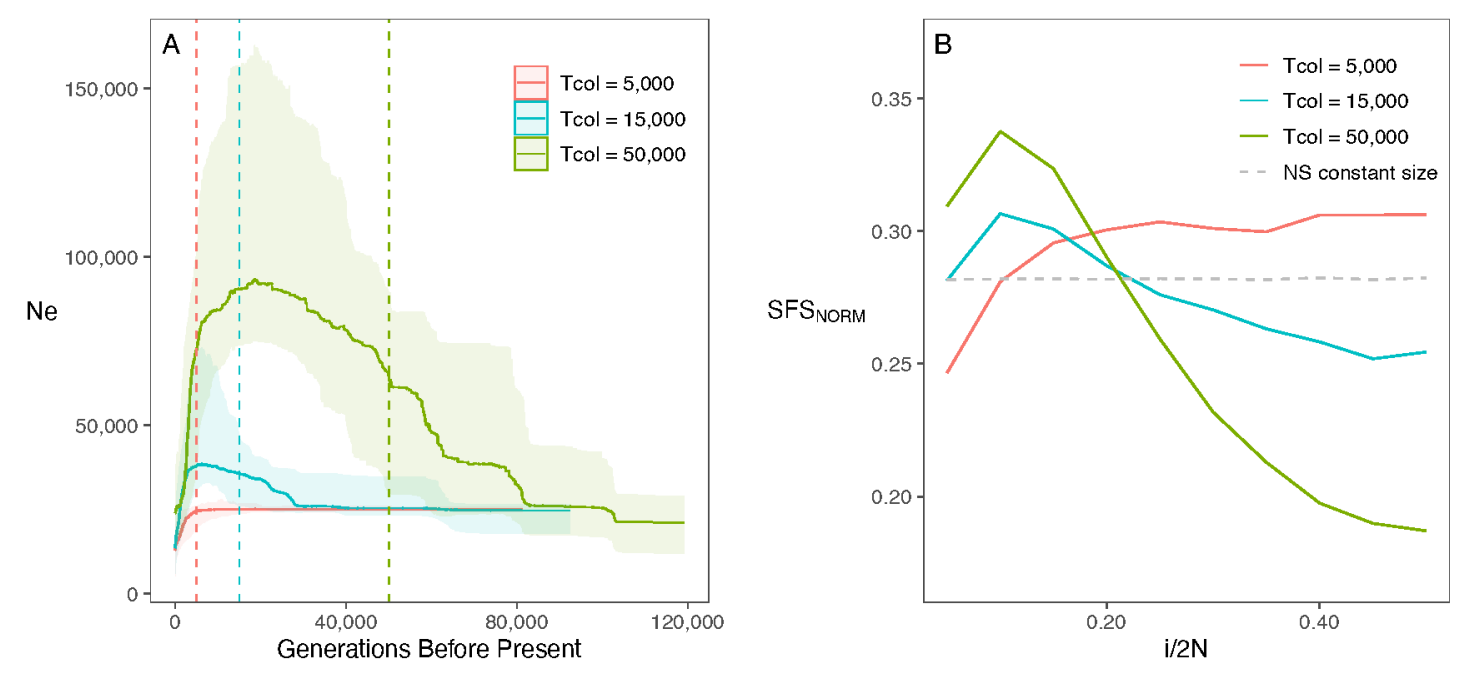
Molecular Ecology Resources

Page 34 of 35
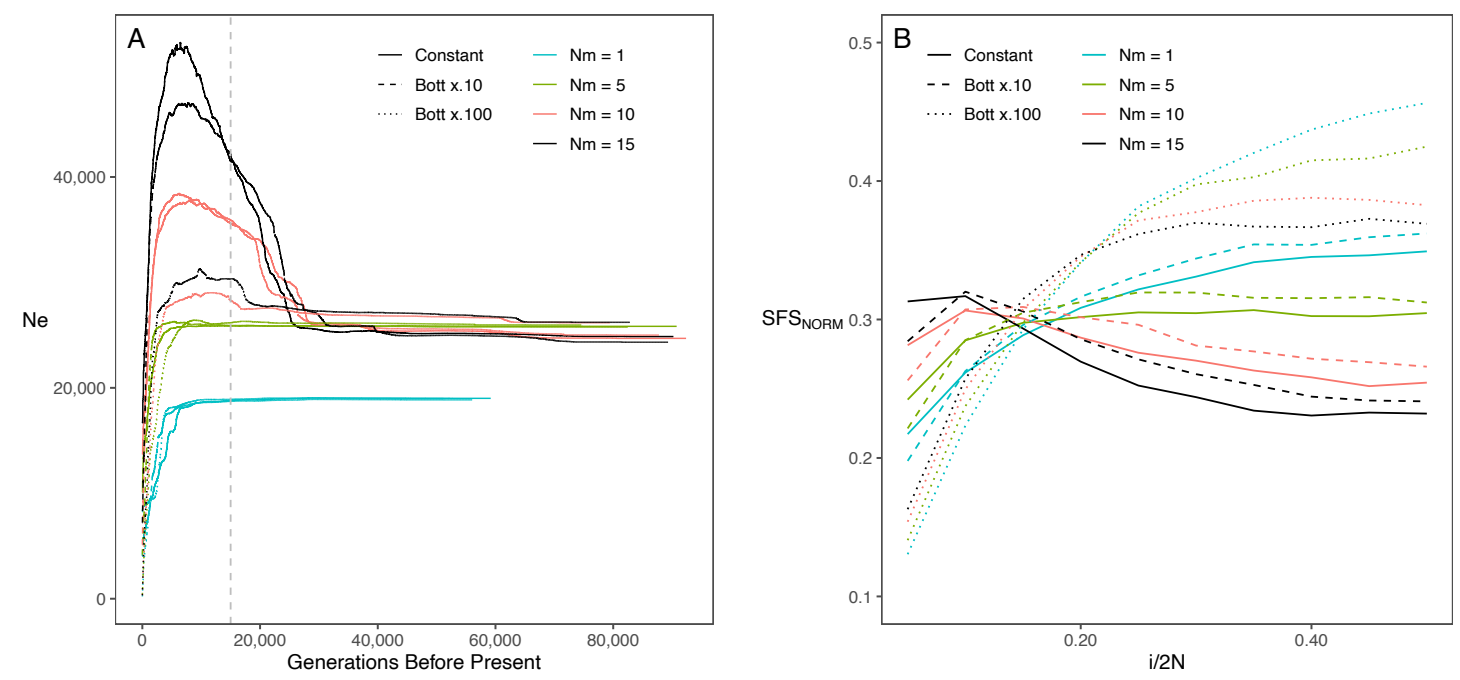

34 


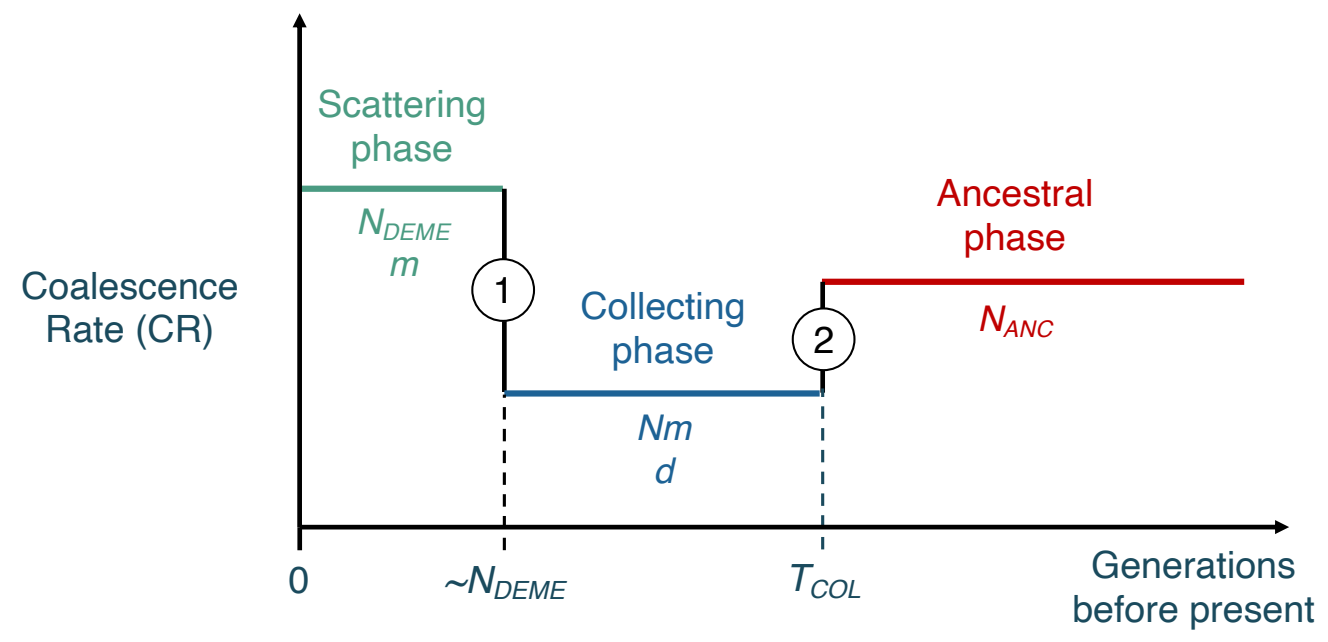

(1) $\Delta_{\mathrm{CR}}<0$ : bottleneck

(2) $\Delta_{C R}>0$ : expansion 